\title{
REAL-TIME MANIPULATION OF MOTION-CAPTURE DATA WITH PATTERN GENERATOR
}

\author{
Shigeru Kuriyama, Yusuke Irino, and Toyohisa Kaneko \\ Toyohashi University of Technology, Department of Information and Computer Sciences \\ Hibarigaoka 1-1, Tenpaku-cho, Toyohasi, Aichi, Japan \\ kuriyama@ics.tut.ac.jp, irino@vcl.ics.tut.ac.jp, kaneko@ics.tut.ac.jp
}

\begin{abstract}
In this paper, a real-time control methodology is proposed for cyclic motion data. A pattern generator is constructed by connecting oscillators composed of mutually inhibited neurons. The rhythmic signal of the pattern generator drives a proportional derivative controller that determines the joint angles of a virtual character, and the signal of the controller is fed back to the oscillator by which the physical parameters of the controller are utilized for stably and smoothly controlling the signals. The motions of bodily components can be autonomously synchronized by mutually feeding their oscillator's state signals. The controller's signals are converted so as to closely approximate those obtained by the motion-capture data by which real-time, interactive, and on-the-fly controls of the motion data are obtained.
\end{abstract}

Keywords: Motion control, captured data, pattern generator, neural oscillator

\section{Introduction}

In creating interactive games, movements of the virtual characters are controlled by instantaneously playing back motion data according to the player's manipulations (usually by pushing the buttons of game-controllers), and motion data are often obtained by capturing real human performances. This type of mechanism controls human motions by triggering predefined behaviors, and is therefore suited to the interactive environments that require rapid responses of the virtual characters, as in sports or battle simulations. However, the expressiveness of the behaviors very much depends on the number of saved motion sequences, and repeated fixed patterns of the replayed motions often damage the sense of reality because natural human movements include chaotic behaviors.

The original version of this chapter was revised: The copyright line was incorrect. This has been corrected. The Erratum to this chapter is available at DOI: 10.1007/978-0-387-35660-0_65 
Intelligent controls of motion-capture data can efficiently generate rich content for interactive playing, since a variety of behaviors are automatically computed from a small amount of data along with some physical rules. The existing technologies for manipulating motion-capture data supply an offline environment for adjusting movements, for example, by a signal-based approach [1], or by physically-based transformations [2]. Although these methods make complicated dynamics controllable while maintaining the naturalness of sampled motions, their space-time optimizations require the manual setting of initial parameters and are therefore unsuitable for real-time and autonomous controls. Blending method of plural data with basis functions [3] also restricts the patterns of composed motions to those of sampled motions, and thus lacks the flexibility to automatically adapt motions under various conditions.

On the other hand, dynamical simulation is suitable for on-the-fly adaptations of motions, and many control methods [4] have been proposed for generating physically plausible motions by producing joint torques with proportional derivative control. Thus, musculo-skeletal segments are feed-forward controlled by a finite state machine that indirectly specifies desirable spacetime states of cyclic motions. Such a control methodology has the flexibility to allow arbitrary poses and timings as controllable states, and can be controlled in an interactive way [5]. However, natural motions require skillful designers to elaborately tune many control parameters, and a pose-based control methodology is unsuitable for globally changing behaviors.

We have already proposed a real-time control [6] on the basis of a pattern generator whose nonlinear dynamics are generated via a pair of mutually inhibited neurons. Our pattern generator can flexibly control cyclic motions in a common mechanism with a small number of parameters, and can smoothly bridge the transition while maintaining overall coordination. Such features enhance the flexibility of hard-wired oscillators [7] and the controllability of pure dynamic simulation [8]. However, our control methodology often loses naturalness in the resulting motions in exchange for its simplified control mechanism. Therefore, this paper proposes a way of constructing adjustable controllers for motion-capture data, by which natural motions can be interactively and smoothly manipulated in real-time.

\section{Pattern Generator}

Our pattern generator uses a neural oscillator proposed by Matsuoka [9] (see Figure 1). It consists of two excited neurons that are linked reciprocally through inhibitory connections, and each neuron has a self-inhibitory effect. The $i$-th oscillator is formulated by two differential equations as

$$
y_{i}=\left[x_{i 1}\right]^{+}-\left[x_{i 2}\right]^{+}, T_{x} \frac{d x_{i j}}{d t}=-x_{i j}-k_{a} a_{i j}+c_{i j}-e_{i j}
$$




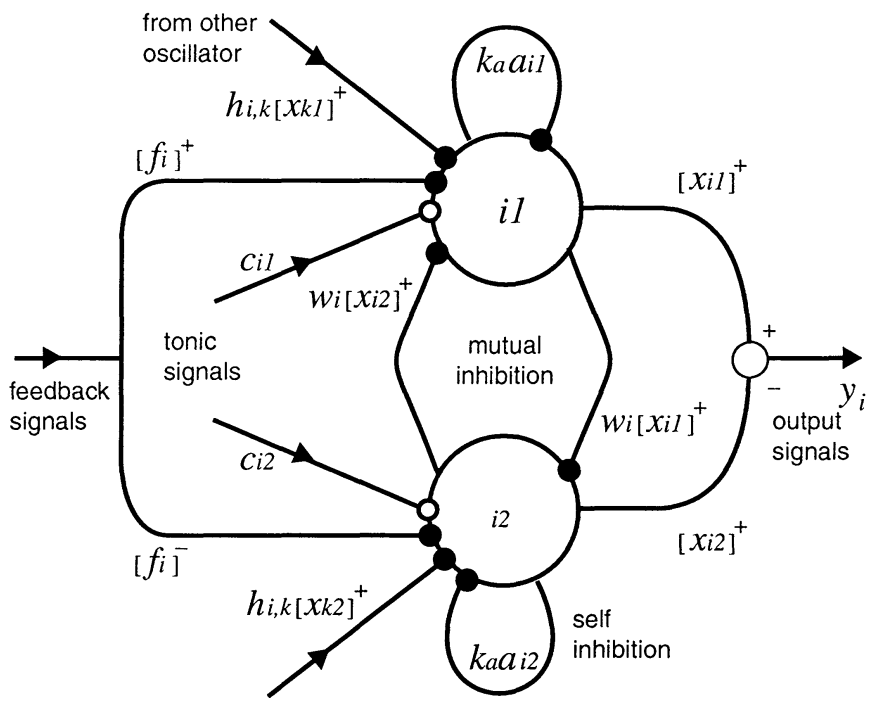

Figure 1. Structure of neural oscillator.

$$
\begin{aligned}
T_{a} \frac{d a_{i j}}{d t} & =-a_{i j}+\left[x_{i j}\right]^{+}, j=1,2 \\
e_{i 1} & =w_{i}\left[x_{i 2}\right]^{+}+\sum_{k \neq i} w_{i, k}\left[x_{k 1}\right]^{+}+h_{f}\left[f_{i}\right]^{+}, \\
e_{i 2} & =w_{i}\left[x_{i 1}\right]^{+}+\sum_{k \neq i} w_{i, k}\left[x_{k 2}\right]^{+}-h_{f}\left[f_{i}\right]^{-}
\end{aligned}
$$

where $y_{i}$ is the neural output, $x_{i j}$ denotes the firing rate, $a_{i j}$ is the degree of adaptation (or self-inhibition) whose effect is controlled by the coefficient $k_{a}$, and $[x]^{+}=\max (x, 0),[x]^{-}=\min (x, 0)$. A pair of neurons, denoted by subscripts $i 1$ and $i 2$, mutually inhibit their firing rates with the connecting weight $w_{i}$. The firing rates from other pairs, denoted by $x_{k \neq i}$, are supplied with the inhibitory connecting weight $w_{i, k}$ in order to coordinate with other oscillators, and a feedback signal $f_{i}$ is also supplied. An external input $c_{i j}$ represents the tonic excitation that controls the magnitude of the output $y_{i}$. Our method uses the tonic $c_{i j}$ for controlling cyclic signals, and other parameters of the oscillator are fixed at empirical values. 


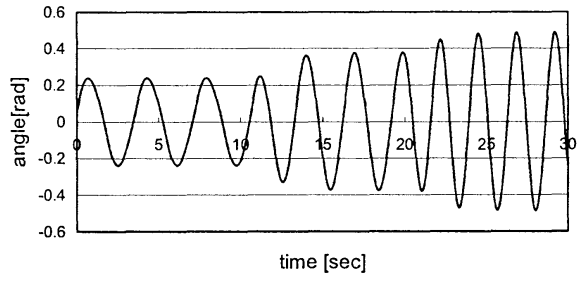

(a) Change in frequency

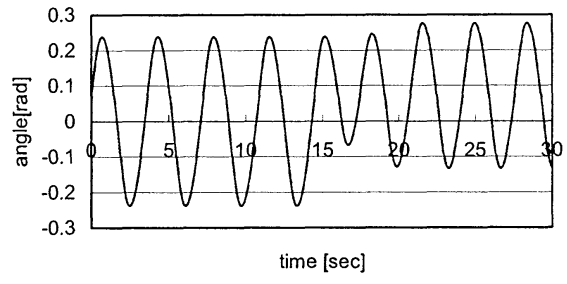

(b) Change in amplitude

Figure 2. Controls of angular positions.

\section{Adjustable Controllers}

\subsection{PD control}

Most controller-based methods use proportional-derivative controls, and we use a similar model by regarding the neural output $y_{i}$ as the rest position of the controller as follows:

$$
\frac{d \omega_{i}}{d t}=k_{i}\left(y_{i}-\theta_{i}\right)-b_{i} \omega_{i}
$$

where $k_{i}$ and $b_{i}$ denote the stiffness and damping parameters of the $i$-th controller. The resonant frequency of the controller, which is approximately proportional to $\sqrt{k_{i}}$, entrains the frequency of the feedback signal $\theta_{i}$, if it is higher than the natural frequency of the oscillator determined from $T_{x}$ and $T_{a}$. Therefore, the stiffness $k_{i}$ is uniquely determined by giving the desired frequency of the control signal, and the ratio between the damping and stiffness is empirically determined to ensure stable oscillations. The angular position is fed to the corresponding oscillator as the feedback signal, $f_{i}=\theta_{i}$, through the inhibitory connection. With this feedback mechanism, the frequency and phase of the oscillator are entrained by the physical property of the controller determined from $k_{i}$ and $b_{i}$ [10].

Figure 2 shows the variation in the angular position: (a) indicates the change in frequency, at which the stiffness and damping are doubled after 10 seconds then doubled again after 20 seconds, and (b) shows the change in amplitude caused by asymmetrically altering the tonic $c_{i 1}=c_{i 2}=1.0$ to $c_{i 1}=1.2, c_{i 2}=$ 0.8 after 15 seconds. These examples show that the signals produced by the neural oscillators have smooth shapes in transitions during sudden variations in control parameters. 


\subsection{Signal conversion}

Since the actual signals for a human's joint angles are hard to obtain with the above control mechanism alone, we introduce a conversion mechanism for angular positions to imitate the waveform of motion-capture data. We first determine the value of the stiffness parameter $k_{i}$ so that the frequency of the angular position $\theta_{i}$ coincides with those of the motion data. Next, the signals generated at the controller are distributed in multiple modes. The $h$-th mode signal, denoted by $\mu_{i}^{h}$, is composed by blending the angular $\theta_{i}$ and velocity $\omega_{i}$ signals with a phase control parameter $\phi_{i}^{h}$ as,

$$
\mu_{i}^{h}=\theta_{i} \cos \left(\phi_{i}^{h}\right)+\frac{\omega_{i}}{\sqrt{k_{i}}} \sin \left(\phi_{i}^{h}\right)
$$

then the conversion is given by

$$
q_{i}=\alpha_{i}^{0}+\sum_{h=1}^{N_{h}} \alpha_{i}^{h} \sin \left(h \mu_{i}^{h}-\beta_{i}^{h}\right)
$$

where $q_{i}$ denotes the joint angle corresponding to the $i$-th controller. The phase $\phi_{i}^{h}$, the coefficients $\alpha_{i}^{h}$, and delay parameters $\beta_{i}^{h}$ are simultaneously computed to minimize the sum of the squared differences between motion-capture signals and the resulting waveform $q_{i}$, where these values are sought in the secondorder approximation $\left(N_{h}=2\right)$. It is noteworthy that the accuracy of an approximation can be arbitrarily improved by increasing the order $N_{h}$, but the higher frequencies produced as a by-product tend to cause unnatural bodily vibrations. Moreover, human visual perception is usually insensitive to the high-frequency component of movements. Furthermore, motion-capture data inevitably include noise components produced in measuring and transforming the data. For these reasons, we experimentally found that the second order approximation effectively balances accuracy with smoothness for our locomotive examples.

\subsection{Implementation}

We attach the adjustable controllers to the joints of a virtual human at the knees and hips of both legs, the pelvis, and the shoulders of both arms. The state signals of an oscillator $x_{k 1}, x_{k 2}$ are supplied through a mutual connection with the weights $w_{i, k}$ as: 1) between left and right hips; 2) from hips to knees on the same side; 3 ) from right shoulder and left hip to left shoulder (and their symmetrical relation); and 4) from left and right hips to pelvis. All joints are assumed to have a hinge-type construction; the degree of freedom is fixed to one, and the joint angles of the elbow are computed in proportion to those of the shoulder on the same side. 
Among three numerical methods, simplex method, simulated annealing, and genetic algorithm, we found that the genetic algorithm stably detects good solutions, whereas the other two methods often fail to do so. The computational cost of the genetic algorithm takes a CPU time of about $200 \sim 400$ seconds for each joint when the time is measured on an Intel Pentium III $650 \mathrm{MHz}$. Although this computation can be pre-processed, we are currently improving this numerical module so as to find solutions in a shorter time. Computation of the converted signal (including the state updates of oscillator and controller) takes a CPU time of only about several microseconds for each joint, and it is linear in relation to the number of joints, which can allow real-time controls. Figure 3 shows the waveforms of hip and knee joints in walking and marching before and after the conversion with adjusted controllers, and Figure 4 shows the resulting motions generated by the conversions.

\section{On-the-fly Controls of Motion Data}

The stiffness of the controller affects the frequency of periodic signals; increasing the value merely speeds up the motion without changing the styles. However, the amplitude of the angular position is also changed according to variations in the stiffness, which often causes distortion of the resulting motions. Therefore, the tonic should be slightly changed to cancel such a side effect. The intrinsic nature of the oscillators ensures the smoothness and stability of the transitional states for joint angles even during a sudden change in

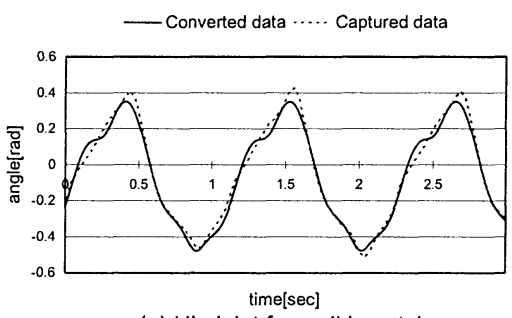

(a) Hip joint for walking style

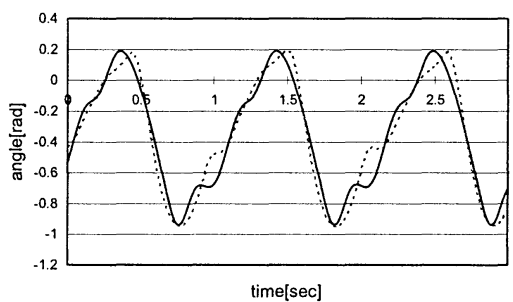

(c) Hip joint for marching style

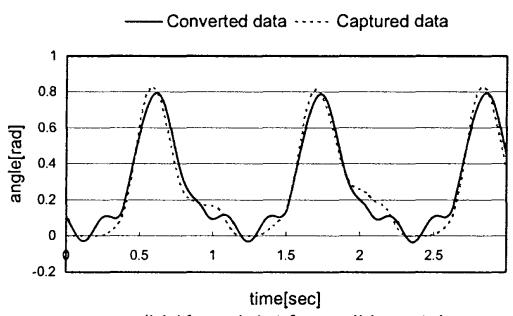

(b) Knee joint for walking style

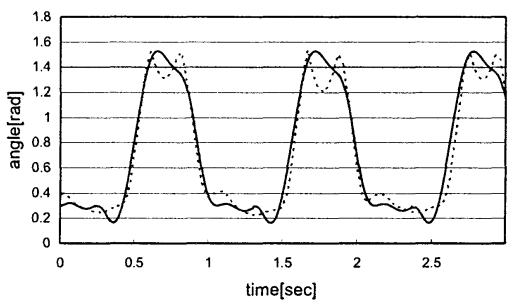

(d) Knee joint for marching style

Figure 3. Parameter fitting for adjustable controllers. 


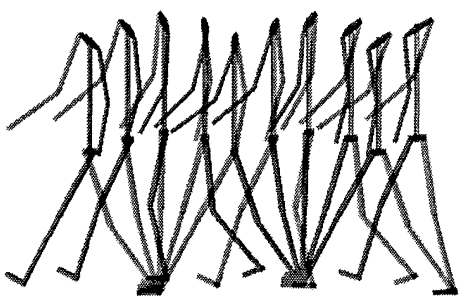

(a) Walking style

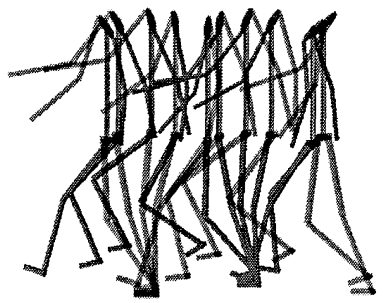

(b) Marching style

Figure 4. Locomotive motions generated through adjustable controllers.

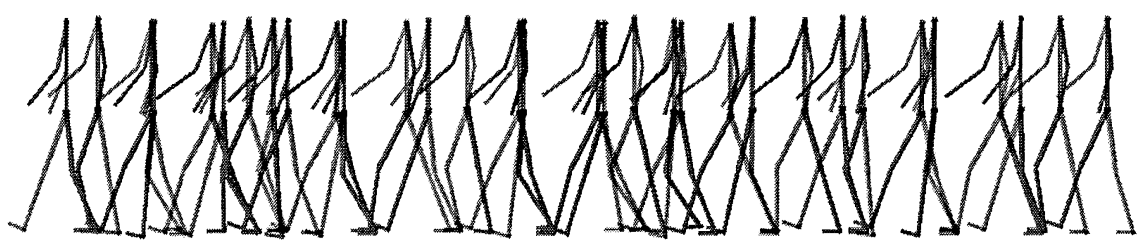

(a) Tonic change for hip joints

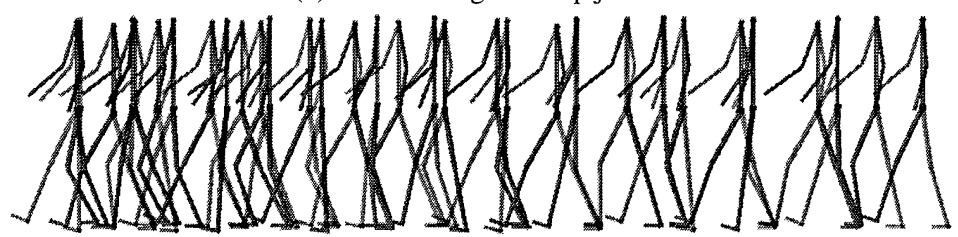

(b) Tonic change for knee joints

Figure 5. Behavior control by changing the tonic of a neural oscillator.

these parameters. However, the motions controlled by the tonic alone are not always natural, so subtle tonic adjustments are therefore required for maintaining plausible movements. Figure 5 demonstrates the manipulation of walking motion in Figure 4 (a) by varying the tonic at the hip joints from $c_{i 1}=c_{i 2}=$ 1.0 to $c_{i 1}=2.4, c_{i 2}=1.2$, and (b) at the knee joints from $c_{i 1}=c_{i 2}=1.0$ to $c_{i 1}=0.9, c_{i 2}=1.5$, where both variations are triggered at the ninth figure.

Moreover, the variables of the adjustable controller could be switched for changing behavior on the fly. However, since a sudden change in their values often generates unnatural movement transitions, a gradual change using some interpolation technique is preferable.

\section{Summary}

Existing methods for editing motion data require time-consuming optimization and careful parameter setting, both of which are unsuitable for real-time manipulations; a deficiency we overcame by adapting a pattern generator to 
motion data while preserving simple control mechanisms. Our concise control strategy is suitable for real-time and on-the-fly manipulations of cyclic motions, with natural variations in cyclic motions intentionally controlled by stochastically fluctuating the stiffness or tonic. Our controller approximately replicates complicated human motions by non-linearly converting the output signal of the controller to imitate motion-capture data. However, it remains unclear whether such a conversion mechanism is suitable for arbitrary types of cyclic motions. This should be clarified by testing many examples; not only for locomotive patterns but also any type of rhythmic motion such as swimming, skiing, or dancing.

\section{References}

[1] M. Gleicher, "Retargeting motion to new characters", In Proceedings of SIGGRAPH '98, pp.33-42, 1998.

[2] Z. Popovic, A. Witkin, "Physically Based Motion Transformation", In Proceedings of SIGGRAPH '99, pp.11-20, 1999.

[3] C. F. Rose, M. F. Cohen, and B. Bodenheimer, "Verbs and adverbs: Multidimensional motion interpolation", IEEE Computer Graphics \& Applications, vol.18, no.5, pp.32-40, 1998.

[4] J. K. Hodgins, P. K. Sweeney, and, D. G. Lawrence, "Generating Natural-looking Motion for Computer Animation", In Proceedings of Graphics Interface '92, pp.265-272, 1992.

[5] J. F. Laszlo, M. van de Panne, and E. Fiume, "Interactive Control For Physically-Based Animation", In Proceedings of SIGGRAPH 2000, pp.201-208, 2000.

[6] S. Kuriyama, Y. Kurihara, and T. Kaneko: "Adaptive Gait Generation via Physiological Controls", Computer Animation 2001, pp.42-51, 2001.

[7] M. McKenna and D. Zeltzer, "Dynamic Simulation of Autonomous Legged Locomotion", In Computer Graphics (SIGGRAPH '90), vol.24, no.4, pp.29-38, 1990.

[8] G. Taga, "A model of the neuro-musculo-skeletal system for human locomotion: I. Emergence of basic gate and II Real-time adaptability under various constraints ", Biological Cybernetics, vol.73, pp.97-121, 1995.

[9] K. Matsuoka, "Sustained Oscillations Generated by Mutually Inhibiting Neurons with Adaptation", Biological Cybernetics, vol.52, pp.367-376, 1985.

[10] S. Schaal, D. Sternad, "Programmable pattern generators", In Proceedings of ICCIN'98, pp.48-51, 1998. 


\section{ENTERTAINMENT ROBOTS \& PHYSICAL SYSTEMS}

